\title{
Reserva funcional renal mediante la depuración de ácido dietilen-triamino-pentaacético marcado con tecnecio-99m en niños que padecieron síndrome urémico-hemolítico
} Renal functional reserve in children with a history of hemolytic uremic syndrome
through technetium-99m diethylene-triamine-penta-acetic acid clearance

\author{
Dr. Guillermo O.A. Bruno ${ }^{a}$ Dra. Stella Maris Diéguez y Dr. Luis E. Voyerc
}

\section{RESUMEN}

Normalmente, la ingesta de carga proteica aumenta el filtrado glomerular, lo que implica reserva funcional renal. Cuando disminuye el número de nefronas pueden observarse filtrados glomerulares normales por hiperfiltración, lo que implica pérdida de reserva funcional, temprano indicador de daño renal y, probablemente, factor contribuyente a su progresión. Determinamos el filtrado glomerular mediante la curva de desaparición plasmática del ácido dietilen-triamino-pentaacético marcado con tecnecio- $99 \mathrm{~m}$, en pacientes con proteinuria y depuración de creatinina normal, que padecieron síndrome urémico-hemolítico con antelación mayor de 1 año. En 33 niños, de 2-16 años de edad, se determinó la reserva funcional renal mediante filtrado glomerular basal y tras carga proteica. Considerando normal un aumento superior al $20 \%$, 16 pacientes mostraron no tener reserva funcional renal.

Esta prueba, por su seguridad y fácil realización, resulta particularmente útil en pacientes que necesitan especial control y tratamiento.

Palabras clave: síndrome urémico-hemolítico, reserva funcional renal, depuración $99 m T c-D T P A$.

\section{SUMMARY}

Protein loads in normal subjects increase glomerular filtration rate (GFR), which implies a renal functional reserve (RFR). Patients who have suffered a loss in the number of nephrons may show normal values of GFR due to hyperfiltration of remnant nephrons, with subsequent loss of RFR. This could be an early sign of renal damage, and probably a contributory factor to renal damage progress.

The objective of this study is to determine the RFR through technetium- $99 \mathrm{~m}$ diethylene-triamine-penta-acetic acid $(99 \mathrm{~m}$ Tc-DTPA) clearance in patients who have recovered from hemolytic uremic syndrome.

a. Medicina Nuclear. Hospital General de Niños "Pedro de Elizalde", Buenos Aires.

b. Nefrología, Departamento de Pediatría. Hospital General de Agudos "Dr. T. Álvarez", Buenos Aires.

c. Nefrología. Hospital General de Niños "Pedro de Elizalde", Buenos Aires.

Correspondencia:

Dr. Luis E Voyer: drvoyer@email.com

Conflicto de intereses: Ninguno que declarar.

Recibido: 18-5-2011

Aceptado: 7-12-2011
Renal functional reserve was determined in 33 children from 2 to 16 years old, with normal values of proteinuria, serum creatinine and creatinine clearance after over a year of having suffered hemolitic uremic syndrome. For that purpose $99 \mathrm{~m} \mathrm{Tc}$ DTPA clearance was determined in basal condition and following protein load. In 17 patients DTPA clearance increased $20 \%$ or more after protein load compared to basal condition, and they were considered to have normal RFR, a probably index of totally recovered renal function; in the remaining 16 patients the increases were lower than $20 \%$, and were considered to have no RFR, condition that was postulated as a contributing factor to renal damage progress.

There was not significant diferences either in age or basal GFR between both groups.

Being the test easier than inuline clearance and more accurate than creatinine clearance, it proves particularly useful for early diagnosis of patients that need special follow-up and treatment. Key words: hemolytic uremic syndrome, renal functional reserve, 99Tc-DTPA clearance.

http:/ /dx.doi.org/10.5546/aap.2012.60

\section{INTRODUCCIÓN}

En sujetos normales, luego de la ingesta de una carga proteica existe un incremento del filtrado glomerular (FG), lo que implica la existencia de una reserva funcional renal (RFR), definida como la diferencia entre el FG estimulado y el FG basal.

En pacientes que por su enfermedad renal tienen disminución del número de nefronas, el FG puede ser normal a expensas de hiperfiltración con la consiguiente pérdida de RFR. En estas condiciones, el daño renal no es reflejado por el FG y la pérdida de la RFR sería un indicador temprano del daño renal. La situación permanente de hiperfiltración parece, a su vez, ser un factor nocivo que conduce a progresión del daño renal. ${ }^{1,2}$

La depuración de inulina se ha aceptado como el método de referencia para medir la FG, pero dadas sus dificultades técnicas se han utilizado otros métodos; entre ellos, la depuración (clearance) de ácido dietilen-triamino-pentaacético marcado con tecnecio 99m (99m Tc-DTPA), que tiene una buena correlación con la depuración de inulina, ${ }^{3}$ cuya ventaja es no necesitar recolección de orina ni infusión constante del trazador. 
El síndrome urémico-hemolítico (SUH), descripto por Gasser en 1955, es una entidad caracterizada por anemia hemolítica microangiopática, trombocitopenia e insuficiencia renal aguda. ${ }^{4} \mathrm{Co}-$ rresponde a Gianantonio y cols., ${ }^{5}$ en 1962 la primera descripción completa de la entidad clínica y de su evolución. El compromiso lesional es predominantemente renal, pero puede afectar también otros parénquimas, como el sistema nervioso central, intestino, páncreas, etc.

La mayoría de los niños supera la etapa aguda y alcanza valores normales de FG, pero algunos de ellos, que sufrieron daño secuelar, lo hacen por hiperfiltración de nefronas residuales, lo que se manifiesta por pérdida de RFR.

\section{OBJETIVOS}

El propósito de este estudio fue determinar la RFR, en pacientes recuperados de la etapa aguda del SUH, midiendo el FG basal y con carga proteica en días separados mediante la curva de desaparición plasmática del 99mTc-DTPA.

\section{POBLACIÓN Y MÉTODOS}

Se estudiaron 33 niños derivados del servicio de Nefrología, 18 masculinos y 15 femeninos, con antecedente de haber padecido SUH con antelación mayor de 1 año y que, al momento del estudio, presentaban valores normales ${ }^{6}$ de: proteinuria, en todos los casos menor de $4 \mathrm{mg} / \mathrm{m}^{2}$ / hora e índice proteína/creatinina con intervalo de 0,03-0,18; de creatinina plasmática, inferior de 0,7 $\mathrm{mg} / \mathrm{dl}$ en niños y de $1 \mathrm{mg} / \mathrm{dl}$ en adolescentes y de depuración de creatinina, intervalo de 80-140 ml/ minuto $/ 1,73 \mathrm{~m}^{2}$ de superficie corporal.

La edad promedio de los pacientes fue 8 años (entre 2 y 16 años).

El FG fue determinado mediante la depuración plasmática de $99 \mathrm{mTc}$-DTPA según modelo unicompartimental, descripto por Brochner-Mortensen para ácido etilen-diamino-tetra-acético marcado con cromo 51 (51Cr-EDTA), ${ }^{7}$ y por Huttunen y cols., ${ }^{3}$ y Palmer y cols., ${ }^{8}$ para 99mTc-DTPA con las siguientes modificaciones: 4 muestras de plasma y comienzo de las extracciones a partir de las 2 h y $30 \mathrm{~min}$, en lugar de 2 $h$, pues encontramos que la pendiente de la curva de actividad plasmática en función del tiempo no era asintótica, lo que sugería no haber alcanzado el equilibrio en el volumen de distribución.

Se determinó la FG basal en condiciones de hidratación normal y con ingesta proteica de acuerdo a edad y sexo según recomendaciones sobre fármacos y alimentos (RDA) de la Academia Na- cional de Ciencias de EE.UU. desde 7 días anteriores a la prueba. Se administró por vía endovenosa entre 50-300 $\mu \mathrm{Ci}(1,85-11,1 \mathrm{MBq})$ de 99mTc-DTPA a las 8 h. A las 2 h y 30 min de la inyección endovenosa, en que se consideró establecido el equilibrio de compartimentos (intravascular y extravascular), se extrajeron 4 muestras de sangre de vena diferente a la de inyección, cada 20-30 min con tiempos rigurosamente cronometrados.

La radioactividad en plasma se midió en un contador gamma con cristal de pozo y el FG fue calculado como VD x K, donde VD es el volumen de distribución del radiofármaco y $\mathrm{K}$ es su tasa de desaparición plasmática.

El resultado fue normalizado a $1,73 \mathrm{~m}^{2}$ de superficie corporal de acuerdo a la fórmula de Haycock y cols. ${ }^{9}$

\section{Prueba de carga proteica}

Entre 7 y 15 días de la prueba basal, se repitió la prueba con carga proteica.

A las 8 h se inyectó el radiofármaco y a los 30 min comenzó la ingesta proteica de $45 \mathrm{~g} / \mathrm{m}^{2}$ de SC, como carne roja cocida, que se cumplió antes de $30 \mathrm{~min}$. Transcurridos $90 \mathrm{~min}$ de finalizada la ingesta, a las 2 h y 30 min de la administración endovenosa del fármaco, se continuó con la prueba en igual forma que la prueba basal.

El trabajo fue previamente aprobado por el Comité de Bioética del Hospital General de Niños "Dr. Pedro de Elizalde". El consentimiento informado por escrito fue entregado y aceptado por los padres y acorde a la edad de los pacientes se explicaron nuevamente los pasos durante la ejecución de la prueba.

\section{Análisis estadístico}

Se aplicó la prueba t para los datos pareados (FG basal y tras carga proteica) y no pareados (valores de los pacientes del grupo 1, sin RFR, comparados con los de pacientes del grupo 2, con FRF) y la prueba de la $\chi^{2}$ para la variable cualitativa (sexo). Se consideraron estadísticamente significativas las diferencias con $p<0,05$. Programa Instat 3.

\section{RESULTADOS}

Se realizaron 33 pruebas completas (en condición basal y con carga proteica) en los 33 niños.

Según las diferencias registradas en el FG entre las dos pruebas, los pacientes se dividieron en dos grupos (Tabla 1): con RFR diecisiete pacientes, 11 masculinos y 6 femeninos, cuando los aumentos fueron de $20 \%$ o más del FG tras la carga pro- 
teica en relación al FG basal y sin RFR dieciséis pacientes, 7 masculinos y 9 femeninos, cuando los aumentos fueron menores del $20 \%$.

Las diferencias de género entre ambos grupos no fueron significativas, $\chi^{2}: 0,2$. Tampoco fueron significativas diferencias de edad, t 0,19, ni de FG basal t 0,25 entre los dos grupos.

Las diferencias en mililitros $(\mathrm{ml})$ de aumento, t 5,78 y su porcentual, $t$ 6,12 entre el grupo 1 y el grupo 2 , fueron significativas $\mathrm{p}<0,05$ y altamente significativas $p<0,001$, respectivamente.

\section{DISCUSIÓN}

Si bien la depuración de inulina es el método patrón para la determinación del FG, su técnica es dificultosa para ser aplicada como método de rutina, y la depuración de creatinina endógena puede sobreestimar el FG en patología renal, como lo demostraron Leville y cols. ${ }^{10}$

El 99mTc-DTPA se elimina por filtrado glomerular $y$, aunque se une en pequeña proporción con las proteínas plasmáticas, existe buena correlación con la depuración de inulina, ${ }^{3}$ con la ventaja de no necesitar recolección de orina ni infusión continua, hecho muy importante en niños; además, el 99mTc-DTPA es fácilmente disponible en los laboratorios de medicina nuclear.

La dosis de radiación absorbida para el $99 \mathrm{mTc}$-DTPA es de $0,016 \mathrm{rad} / \mathrm{mCi}$ para cuerpo entero y $0,555 \mathrm{rad} / \mathrm{mCi}$ para vejiga, asumiendo una retención indefinida; esta exposición a la radiación es relativamente pequeña con la dosis administrada. ${ }^{3}$

Para considerar evidencia de RFR se asumió un incremento de $20 \%$ o más en FG con carga proteica, dos veces el valor de la fluctuación diurna, que es aproximadamente $10 \%$ del FG medio. ${ }^{11}$

Carlsen y cols. ${ }^{12}$ compararon las depuraciones plasmáticas de cuatro preparados comerciales distintos de DTPA con la depuración de Cr51EDTA y observaron que tres de estos preparados subestimaron en diverso grado el FG, diferencias probablemente debidas a diferentes ligaduras o "binding" con las proteínas.

En nuestros estudios comparamos diferencias entre el FG basal y el FG con carga proteica utilizando las mismas partidas e idéntico origen (Comisión Nacional de Energía Atómica, CNEA) de DTPA y en las mismas condiciones de hidratación, alimentación previa y horarios de comienzo para las pruebas, basal y con carga proteica.

Durante la ejecución del método se encontró un error al referir el FG por superficie corporal según el nomograma utilizado, ${ }^{13}$ error que debe ser evitado. Para todas las determinaciones empleamos la fórmula de Haycock. ${ }^{9}$

Este estudio mostró un mayor porcentaje de pacientes sin RFR, con respecto a estudios anteriores. ${ }^{14,15}$ Esto podría deberse a la inclusión de pacientes con menos de dos años de evolución después de haber padecido el episodio agudo de SUH.

La pérdida de RFR es sinónimo de hiperfiltración glomerular en condiciones basales. Estos cambios hemodinámicos serían responsables de la progresión de la enfermedad renal contribuyendo a la nefroesclerosis según la teoría de Brenner, demostrada en experimentos efectuados con animales, ${ }^{1,2}$ y que probablemente ocurren también en estos pacientes según observaciones del seguimiento a largo plazo. ${ }^{16}$ Se sabe que pacientes que padecieron SUH, aunque hayan recuperado el FG en reposo, deben ser controlados regularmente por la posibilidad de aparición tardía de daño renal. Al determinar la RFR, pueden detectarse aquellos con hiperfiltración glomerular.

TABLA 1. Medición del filtrado glomerular (FG) basal y con carga proteica

\begin{tabular}{|c|c|c|c|c|}
\hline \multirow[t]{2}{*}{ Variables* } & \multicolumn{4}{|c|}{ Pacientes } \\
\hline & \multicolumn{2}{|c|}{$\begin{array}{c}\text { Con RFR } \\
n=17\end{array}$} & \multicolumn{2}{|c|}{$\begin{array}{c}\text { Sin RFR } \\
n=16\end{array}$} \\
\hline Edad (años) & \multicolumn{2}{|c|}{$7,9(4,0)$} & \multicolumn{2}{|c|}{$8,19(4,44)$} \\
\hline FG basal $\left(\mathrm{ml} / \mathrm{min} / 1,73 \mathrm{~m}^{2}\right)$ & $105,5(13,8)$ & $\mathrm{t}: 4,46$ & $101,44(9,66)$ & $\mathrm{t}: 0,63$ \\
\hline FG post Prot $\left(\mathrm{ml} / \mathrm{min} / 1,73 \mathrm{~m}^{2}\right)$ & $137,2(28,9)$ & $\mathrm{p}:<0,001$ & $103,75(9,13)$ & p: NS \\
\hline ml de aumento & \multicolumn{2}{|c|}{$34,7(22,7)$} & \multicolumn{2}{|c|}{$2,31(3,68)$} \\
\hline Aumento (\%) & \multicolumn{2}{|c|}{$33,8(20,7)$} & \multicolumn{2}{|c|}{$2,43(4,05)$} \\
\hline
\end{tabular}

${ }^{*}$ Los resultados se expresan como media (DE).

RFR: reserva funcional renal.

Post Prot: post carga proteica. 


\section{CONCLUSIONES}

De los 33 pacientes con antecedente de SUH estudiados mediante la curva de desaparición plasmática de $99 \mathrm{mTc}$-DTPA en condiciones basales y tras carga proteica, 16 pacientes $(48,5 \%)$ mostraron no tener RFR.

Siendo esta prueba más fácil de efectuar que la depuración de inulina y más segura que la depuración de creatinina, resulta particularmente útil para el diagnóstico de pacientes que necesitan especial control y tratamiento.

\section{Agradecimientos}

A los técnicos Graciela Gesteiro y Fernando Turquía por la asistencia técnica.

A la Comisión Nacional de Energía Atómica por proveer los radiofármacos necesarios.

\section{BIBLIOGRAFÍA}

1. Remuzzi G, Ruggenenti P, Benigni A. Understanding the nature of renal disease progression. Kidney Int 1997;51:25. 11:473-76.

2. Brenner BM, Lawler EV, Mackenzie HS. The hyperfiltration theory: A paradigm shift in nephrology. Kidney Int 1996;49:1774-77.

3. Huttunen K, Huttunen NP, Koivula A, Ahonen A, et al. 99mTc-DTPA a useful clinical tool for the measurement of glomerular filtration rate. Scand J Nephrol 1982;16:237-41.

4. Gasser V, Gautier E, Stecck A, Siebenman R, et al. Hämolytisch-urämische Syndrome. Schweiz Med Wochenschr 1955; 85:905-9.
5. Gianantonio CA, Vitacco M, Mendilaharzu J, Mendilaharzu F, Rutty A. Acute renal failure in infancy and childhood. Clinical course and treatment of 41 patients. J Pediatr 1962;61:660-78

6. Nefrología Pediátrica. $2^{\mathrm{a}}$ ed. Buenos Aires: Sociedad Argentina de Pediatría; 2008. Págs.112 y 806.

7. Brochner-Mortensen J. A simple method for the determination of glomerular filtration rate. Scand J Clin Lab Invest 1972;30:271-4.

8. Palmer E, Scot JA, Strauss HW. Practical nuclear medicine. Filadelfia: W.B. Saunders Company; 1992.Págs.241-73.

9. Haycock G, Schhwartz G, Wisotsky D. Geometric method for measuring body surface area. J Pediatr 1978;93:62-6.

10. Leville M,Hadj-Aissa A, Pozet N, et al. Restrictions on use of creatinine clearance for measurement of renal functional reserve. Nephron 1989;51:233-6.

11. Hostetter TH. Human renal response to a meat meal. Am J Physiol 1986;250:F613-8.

12. Carlsen JE, Moller ML, Lund JO, Trap-jensen J. Comparison of four commercial TC-99m(Sn)DTPA preparations used for the measurement of glomerular filtration rate: concise communication. J Nucl Med 1980;21:126-9.

13. Bruno GOA, Pérez A, Alvarado C, Voyer LE. The nomogram of body surface area induces a systematic error in the determination of the glomerular filtration rate. Pediatr Nephrol 1993;7:776-7.

14. Tufro A, Arrizurieta EE, Repetto H. Renal functional reserve in children with a previous episode of a haemolyticuraemic syndrome. Pediatr Nephrol 1991;5:184-8.

15. Dieguez S, Ayuso S, Brindo M, Osinde E, et al. Renal functional reserve evolution in children with a previous episode of hemolytic uremic syndrome. Nephron Clin Pract 2004;97(3):c118-22.

16. Caletti MG, Gallo G, Gianantonio CA. Development of focal segmental sclerosis and hyalinosis in hemolytic uremic syndrome. Pediatr Nephrol 1996;10:687-92. 